\section{FABRICATION OF LOW-LOSS OPTICAL FIBRES CONTAINING RARE-EARTH IONS}

Indexing term: Optical fibres

Fibres containing rare-earth ions have been produced by an extended MCVD process. The fibres have very high absorption levels in the visible and near infra-red regions, without significantly compromising the low-loss characteristics of the fibre at wavelengths between 950 and $1400 \mathrm{~nm}$.

Introduction: One of the requirements of an optical fibre amplifier or laser is that it should have a high absorption at the pump wavelength and a very low loss at the laser wavelength. Conventionally, this has been achieved by using a high dopant concentration in a short length of neodymium-doped compound-glass ${ }^{1}$ or silica ${ }^{2}$ fibre. However, an attractive alternative approach is to use lower dopant levels and thus exploit the long interaction lengths and very low losses inherent in communications-grade optical fibres.

In optical fibre sensors, a change in the optical properties of rare-earth ions within a glass matrix can be used to measure a number of parameters, particularly temperature. Thus, short lengths $(\sim 3 \mathrm{~cm})$ of heavily doped glass or glass fibre have been used to measure temperature by means of the change in either the absorption spectrum ${ }^{3}$ or fluorescence lifetime. ${ }^{4} \mathrm{~A}$ distributed temperature sensor ${ }^{5}$ based on these effects requires much lower concentrations of dopant, as well as low fibre loss, in order to penetrate a length of several hundred metres.

We report here a novel extension of the MCVD fabrication process which allows the fabrication of both mono- and multimode optical fibres containing rare-earth ions at concentrations of up to $0.25 \mathrm{wt} \%$ in the core region. The technique is unique in that it allows the use of starting materials, e.g. rareearth halides, which have a high melting point $\left(>580^{\circ} \mathrm{C}\right)$ and hence have hitherto been unusable, since they exhibit a very low vapour pressure at the temperatures commonly encountered in reactant delivery systems for optical fibre fabrication.

Initial work has concentrated on the lanthanide series as dopants, particularly neodymium and erbium, as these are of interest for both sensors and lasers. We have produced fibres with very high-loss absorption bands $(>3000 \mathrm{~dB} / \mathrm{km})$ in the visible and near infra-red regions, while maintaining the characteristic low loss $(<2 \mathrm{~dB} / \mathrm{km})$ of the MCVD fabrication process in the 'second window' for optical communications $(1300 \mathrm{~nm})$. The technique may also be used to incorporate many other rare-earth and transition metals into optical fibres.

Fibre fabrication: The fibre preform is fabricated using the MCVD technique, with a number of important modifications to permit the incorporation of further dopants into the core glass. Prior to deposition, a conventional deposition tube is prepared by inserting the required dopant-for example, $\mathrm{NdCl}_{3} 6 \mathrm{H}_{2} \mathrm{O}\left(99.9 \%\right.$ pure, melting point $\left.=758^{\circ} \mathrm{C}\right)$--into a dopant carrier chamber (Fig. 1), where it is dehydrated by heating under a chlorine atmosphere. This step also fuses the

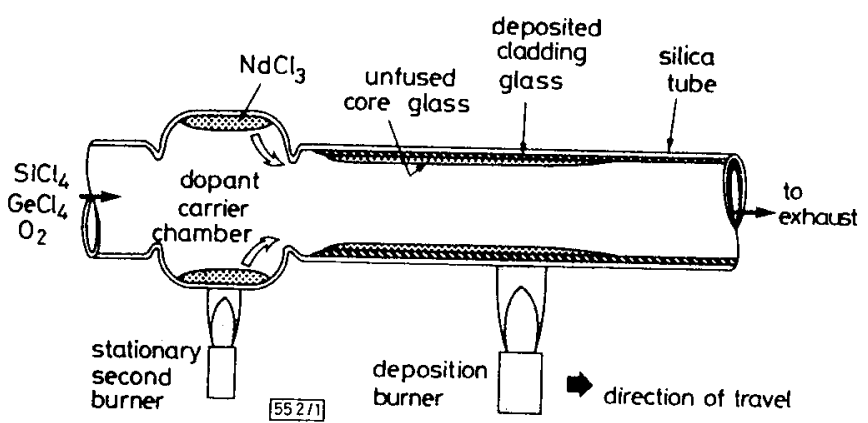

Fig. 1 MCVD process for low vapour-pressure dopants

anhydrous $\mathrm{NdCl}_{3}$ crystals to the chamber wall, thus preventing them from passing down the tube and forming bubbles in the glass subsequently deposited. The inside of the deposition tube is then cleaned by gas-phase etching using $\mathrm{SF}_{6}$ to remove any dopant deposited during the drying process, following which the cladding glass is deposited in the usual manner. During the core deposition, however, the dopant carrier chamber is heated to around $1000^{\circ} \mathrm{C}$ by a stationary second burner to produce small quantities of $\mathrm{NdCl}_{3}$ vapour. The vapour is carried downstream by the reactant flow, where it is oxidised to $\mathrm{Nd}_{2} \mathrm{O}_{3}$ in the hot zone formed by the deposition burner and incorporated into the core.

Initial measurements showed that the first drying stage did not dehydrate the $\mathrm{NdCl}_{3}$ sufficiently to produce low-loss fibres. Consequently, a second drying process was introduced in which the core, consisting of $\mathrm{SiO}_{2}, \mathrm{GeO}_{2}$ and a small amount of $\mathrm{Nd}_{2} \mathrm{O}_{3}$, is deposited unfused at a low temperature. The porous core layer on the inside of the deposition tube is subsequently dried by heating in a chlorine atmosphere, after which it is fused to form a clear nonporous layer. The tube is then conventionally collapsed to form a solid rod and pull into a fibre.

The process is simple to implement on existing MCVD fabrication equipment and gives reproducible fibres in terms of both refractive index profile and dopant concentration. In addition, it may be adapted to incorporate almost any dopant into the core of single- or multimode fibres.

\section{Results and discussion}

(a) $\mathrm{Nd}^{3+}$-doped fibres: Absorption measurements on monoand multimode fibres show that, as expected, neodymium is incorporated into the glass matrix as the trivalent $\mathrm{Nd}^{3+}$ ion. Fibres with absorption levels (at $590 \mathrm{~nm}$ ) ranging from $40 \mathrm{~dB} /$ $\mathrm{km}$ to $30000 \mathrm{~dB} / \mathrm{km}$ (corresponding to dopant levels of 0.3 to 300 parts in $10^{6}$ of $\mathrm{Nd}^{3+}$ ) have been fabricated. The absorption spectrum for a $500 \mathrm{~m}$ length of neodymium-doped fibre having a dopant level of $\sim 30$ parts in $10^{6}$ is shown in Fig. $2 a$. The very high absorption levels in the visible and near infrared regions of up to $3000 \mathrm{~dB} / \mathrm{km}$ can be clearly seen. Despite this high loss, it is remarkable to observe the existence of a low-loss window between 950 and $1350 \mathrm{~nm}$ of $<2 \mathrm{~dB} / \mathrm{km}$, a figure not very different from that observed in conventional fibres. Moreover, we believe the small excess loss is due to increased scattering in the fibre, rather than to the absorptionband tails. The low $\mathrm{OH}^{-}$absorption peak at $1390 \mathrm{~nm}$ indicates the sucess of the techniques used to dry the neodymium compounds, both before and during the deposition.

The fluorescence spectrum of a fibre doped with 300 parts in $10^{6} \mathrm{Nd}^{3+}$ is shown in Fig. $2 b$, where broad fluorescence
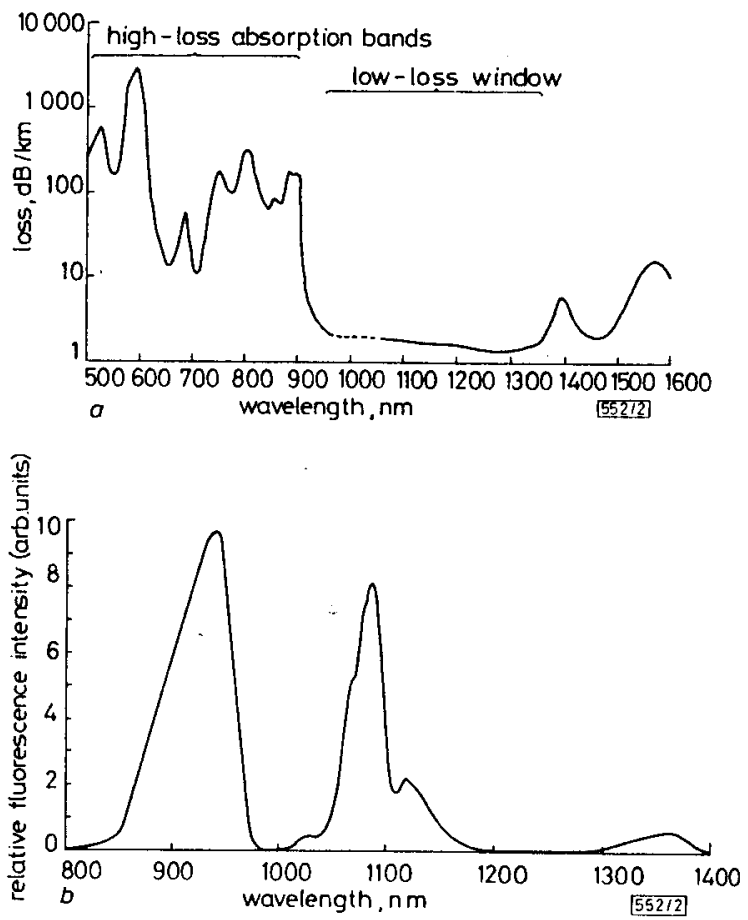

Fig. 2

a Absorption spectrum of fibre containing $\sim 30$ parts in $10^{6} \mathrm{Nd}^{3+}$ $b$ Fluorescence spectrum of fibre containing $\sim 300$ parts in $10^{6}$ $\mathrm{Nd}^{3+}$ 
bands with peak wavelengths of 940,1080 and $1370 \mathrm{~nm}$ can be clearly seen. As a result of the high-silica host glass, the bands are shifted to slightly longer wavelengths than the corresponding bands in compound glasses used for conventional lasers. Measurements of the $1 / e$ fluorescence lifetime using a $590 \mathrm{~nm}$ pump wavelength gave a figure of $450 \mu$ s for both the 940 and 1080 transitions. This is in good agreement with previously published data for bulk silica host material. ${ }^{6}$ In addition, the consistency of the dopant incorporation along the fibre length has been resolved by measuring the local attenuation along the fibre length using an OTDR technique. The source wavelength of $620 \mathrm{~nm}$ is chosen to lie on the tail of the $590 \mathrm{~nm}$ absorption band so as to achieve a manageable attenuation. The results are shown in Fig. 3, and indicate good uniformity of dopant incorporation along the length of the fibre, thus indicating the high degree of control in the fabrication process. The OTDR variations seen are similar to those observed in conventional monomode fibres. ${ }^{7}$

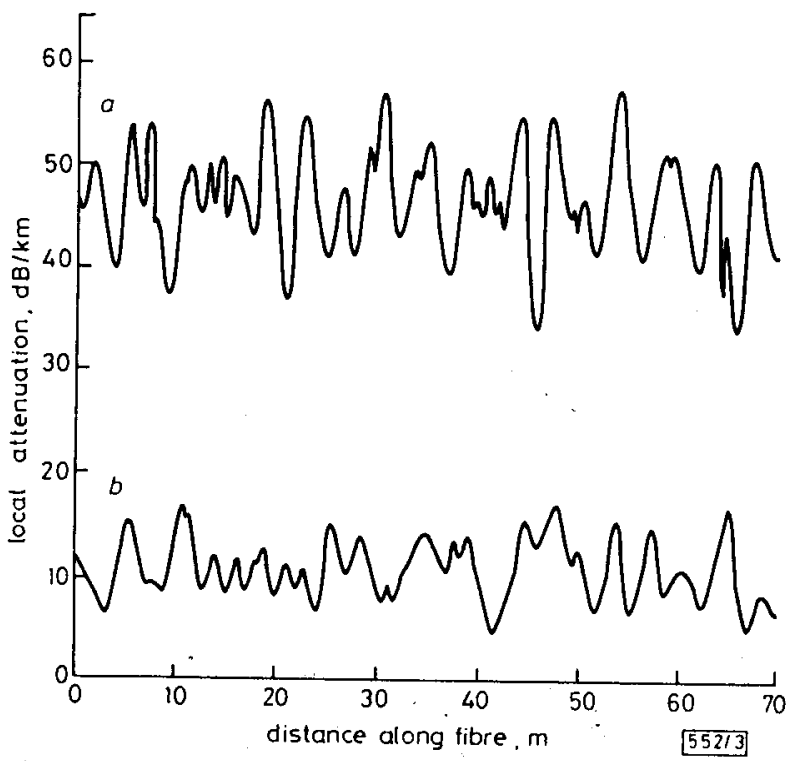

Fig. 3 Local attenuation of single-mode fibres measured using an OTDR technique

a $\mathrm{Nd}^{3+}$-doped fibre

$b$ Reference 'telecomms-type' fibre

(b) Other impurity dopants: It is believed that almost any dopant can be incorporated by the above technique. As an indication of this, the absorption spectrum of a fibre containing $\sim 2$ parts in $10^{6} \mathrm{Er}^{3+}$ is shown in Fig. 4. The result again shows a region of very low loss $(<2 \mathrm{~dB} / \mathrm{km})$, despite heavy absorptions at other wavelengths. Measurements on various fibres pulled from this preform indicate that the rather high $\mathrm{OH}^{-}$absorption peaks seen in the spectrum can be attributed to an inadequate buffer layer thickness, rather than to a deficiency in the drying process. Fibres containing up to $0.25 \mathrm{wt} \% \mathrm{Er}^{3+}$ have been made in which peak absorptions up to $50 \mathrm{~dB} / \mathrm{m}$ have been measured. Even in this case, loss windows of lower than $40 \mathrm{~dB} / \mathrm{km}$ are present. Other rareearth dopants incorporated to date have included $\mathrm{Tb}^{3+}$ and $\mathrm{Eu}^{3+}$.

Conclusions: We have developed a simple, reproducible technique for the uniform incorporation of rare-earth ions into the core of multi- and monomode optical fibres. The fibres have

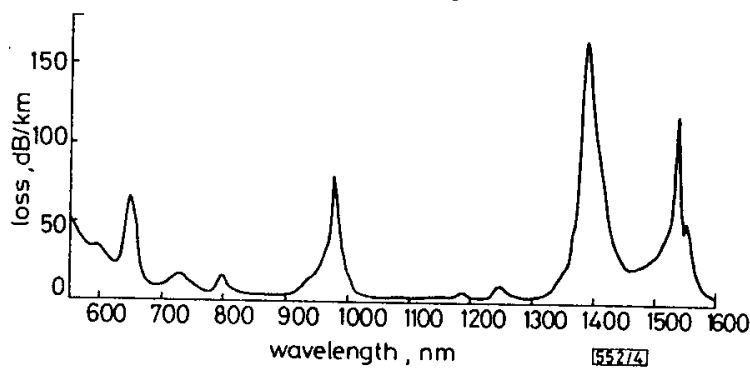

Fig. 4 Absorption spectrum of fibre containing $\sim 2$ parts in $10^{6} \mathrm{Er}^{3+}$ high absorption levels in the visible and near infra-red regions without significantly compromising the low loss characteristics at wavelengths between 950 and $1400 \mathrm{~nm}$. These properties are ideal for use in distributed sensors and as active fibres in optical communication systems.

Acknowledgments: We wish to thank R. J. Mears, M. P. Gold and R. I. Laming for the fluorescence measurements. A Readership was provided by Pirelli General plc (DNP). The work was supported under the JOERS programme.

S. B. POOLE

D. N. PAYNE

15th July 1985

M. E. FERMANN

Department of Electronics \& Information Engineering

The University, Southampton SO9 5NH, United Kingdom

\section{References}

1 SNITzER, E.: 'Neodymium glass laser'. Proceedings of 3rd international congress on quantum electronics, Paris, 1963, pp. 999-1019

2 STONE, J., and BURRUS, C. A.: 'Neodymium doped silica lasers in end-pumped fiber geometry', Appl. Phys. Lett., 1973, 23, pp. 388-389

3. SNITZER, E., MOREY, W. W., and GLENN, w. H.: 'Fiber optic rare earth temperature sensors'. Proceedings of $1 \mathrm{st}$ international conference on optical fibre sensors, London, 1983, pp. $79 m 82$

4 GRATtan, K. T. V., and Palmek, A. W.: 'A simple, inexpensive neodymium rod fibre optic temperature sensor'. Proceedings of 3 rd international conference on optical fibre sensors, San Diego, 1985 , p. 142

5 PAYNE, D. N.: 'Fibres for sensors'. Proceedings of 2 nd international conference on optical fibre sensors, Stuttgart, 1984, pp. 353-360

6 NAMIKAWA, H., ARAl, K., KUMATA, K., ISHI, Y., and TANAKA, H.: 'Preparation of Nd-doped $\mathrm{SiO}_{2}$ glass by plasma torch CVD', $J p n$. J. Appl. Phys., 1982, 21, pp. 1360-1362

7 GOLD, M. P., and HARTOG, A. H.: 'Determination of structural parameter variations in single-mode optical fibres by time-domain reflectometry', Electron. Lett., 1982, 18, pp. 489-490 